\title{
A Case of Dural Arteriovenous \\ Case \\ Report Fistula Involving the Inferior Petroclival Vein
}

Naoki Hayashi, ${ }^{1,2}$ Masahiko Kawanishi, ${ }^{2}$ Shuichi Okubo, ${ }^{1}$ Naohiro Osaka, ${ }^{1}$ Kunihiko Osaka, ${ }^{1}$ and Takashi Tamiya ${ }^{2}$

Objective: We report a very rare case of dural arteriovenous fistula (DAVF) involving the inferior petroclival vein (IPCV). Case Presentation: The patient was a 77-year-old woman. She suffered from right ophthalmalgia, pulsatile tinnitus, blepharedema, and chemosis, as well as right ptosis and diplopia (right oculomotor nerve palsy). Our imaging examinations demonstrated DAVF involving the IPCV. Since the proximal side of the right inferior petrosal sinus (IPS) was obstructed, the main venous drainage flow refluxed retrogradely from the IPS to the cavernous sinus (CS). Therefore, her clinical symptoms were similar to those of CS-DAVF. We successfully performed transvenous coil embolization (TVE) in the IPCV and IPS.

Conclusion: We experienced a very rare case of DAVF involving the IPCV. We believe that it is important in each case to understand the 3D vascular anatomy by making full use of 3D-DSA and other modalities to accurately identify the shunt point and venous drainage structure.

Keywords $>$ dural arteriovenous fistula, inferior petroclival vein, transvenous coil embolization, inferior petrosal sinus

\section{Introduction}

Veins flowing into the anterior condylar confluence (ACC) from the cavernous sinus (CS) include the inferior petroclival vein (IPCV) distributed on the inferior (extracranial) surface of the petroclival fissure (PCF). It is located at a mirror-image of the inferior petrosal sinus (IPS) distributed on the superior (intracranial) surface of the PCF and these communicate with each other., ${ }^{1,2}$

We experienced a patient with very rare dural arteriovenous fistula (DAVF) involving the IPCV. Venous drainage flowed into the IPS and ACC and obstructed the proximal side of the IPS, which caused reflux into the CS and

${ }^{1}$ Department of Neurosurgery, Osaka Neurosurgical Hospital, Takamatsu, Kagawa, Japan

${ }^{2}$ Department of Neurological Surgery, Kagawa University Faculty of Medicine, Kita-gun, Kagawa, Japan

Received: June 4, 2018; Accepted: September 5, 2018 Corresponding author: Naoki Hayashi. Department of Neurosurgery, Osaka Neurosurgical Hospital, 378-1 Sanmyou-cho, Takamatsu, Kagawa 761-8083, Japan Email: nhayashi@osaka-nhp.jp

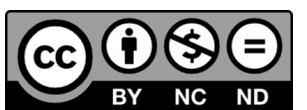

This work is licensed under a Creative Commons Attribution-NonCommercialNoDerivatives International License.

(C)2019 The Japanese Society for Neuroendovascular Therapy manifested mainly ocular symptoms similar to those of CS-DAVF. We succeeded in occluding the DAVF by performing transvenous coil embolization (TVE) to the shunt point of the IPCV and venous drainage route to the IPS.

\section{Case Presentation}

The patient was a 77-year-old female with a past medical history of hypertension and hyperlipidemia with no particular familial medical history.

She noticed right shoulder pain, right ophthalmalgia, and right lacrimation in June 20XX. Since right pulsating tinnitus developed in January 20XX +1 , right ophthalmalgia got worse in early March of the same year, and severe right blepharedema and chemosis and right ptosis developed, she visited a physician and DAVF of the CS was suspected of brain MRI and MRA, for which she was referred to and admitted to our department.

On admission, her consciousness was clear and no cognitive dysfunction was noted. Right oculomotor nerve palsy-associated diplopia and right ptosis were observed. In addition, headache, right ophthalmalgia, right pulsating tinnitus, blepharedema, and chemosis were noted.

On MRI on admission, mild chronic ischemic changes were noted, but no acute change, such as a hemorrhagic 
lesion, cerebral infarction, and brain swelling, was noted. However, dilatation of the cortical veins of the right brain hemisphere was observed on T2-weighted image (T2WI) and susceptibility-weighted imaging (SWI), suggesting cortical venous reflux. High reticular intensities accumulated on the inferior lateral region of the IPS on MRA-time-of-flight (TOF), and the IPS, CS, superior ophthalmic vein (SOV), and superficial middle cerebral vein (SMCV) were visualized as markedly high intensities. Based on these findings, the patient was diagnosed with DAVF with a shunt in adjacent to the ventrolateral region of the IPS and reflux from the IPS to CS (Fig. 1).

\section{Angiography}

Under general anesthesia, a 4Fr regular sheath: Radifocus Introducer II H (Terumo Corporation, Tokyo, Japan) was placed through the right femoral artery, and diagnostic angiography was closely performed using a $4 \mathrm{Fr} \mathrm{CX}$ catheter (Gadelius Medical K.K., Tokyo, Japan). Selective angiography of the left ascending pharyngeal artery (APA) was performed to acquire 3D-DSA and the shunt point was closely investigated three-dimensionally.

The reticular feeder from the left APA accumulated at a site on the inferior surface of the IPS and the contrast medium was most densely visualized, ${ }^{3)}$ suggesting that this was the shunt point. A venous pouch was found on the inferior lateral region of the IPS and blood drained into the IPS. The proximal side of the IPS was obstructed and venous drainage flow which entered the IPS mainly retrogradely drained from the IPS into the CS and then refluxed from the SOV and SMCV into the cortical veins. Moreover, venous drainage into the IPS continued to the ACC and the
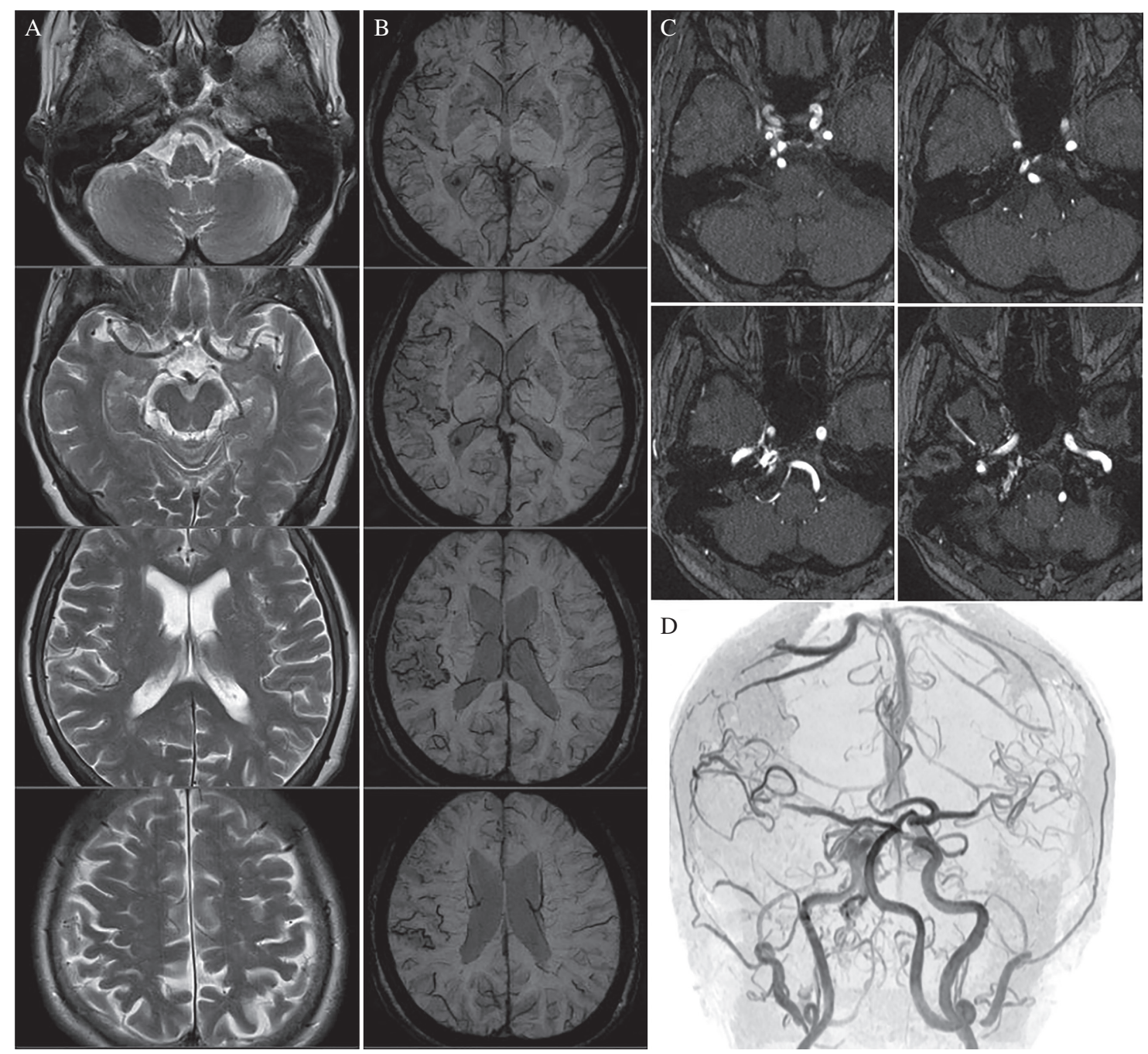

Fig. 1 Brain MRI and MRA at the first visit. MRI (T2WI) does not show brain edema (A), but MRI (SWI) demonstrates the cortical venous reflux at the right cerebral hemisphere (B). MRA (C: MRA-TOF, D: MRA) shows DAVF located near the right petrous apex. DAVF: dural arteriovenous fistula; SWI: susceptibility-weighted imaging; T2WI: T2-weighted image; TOF: time-of-flight 


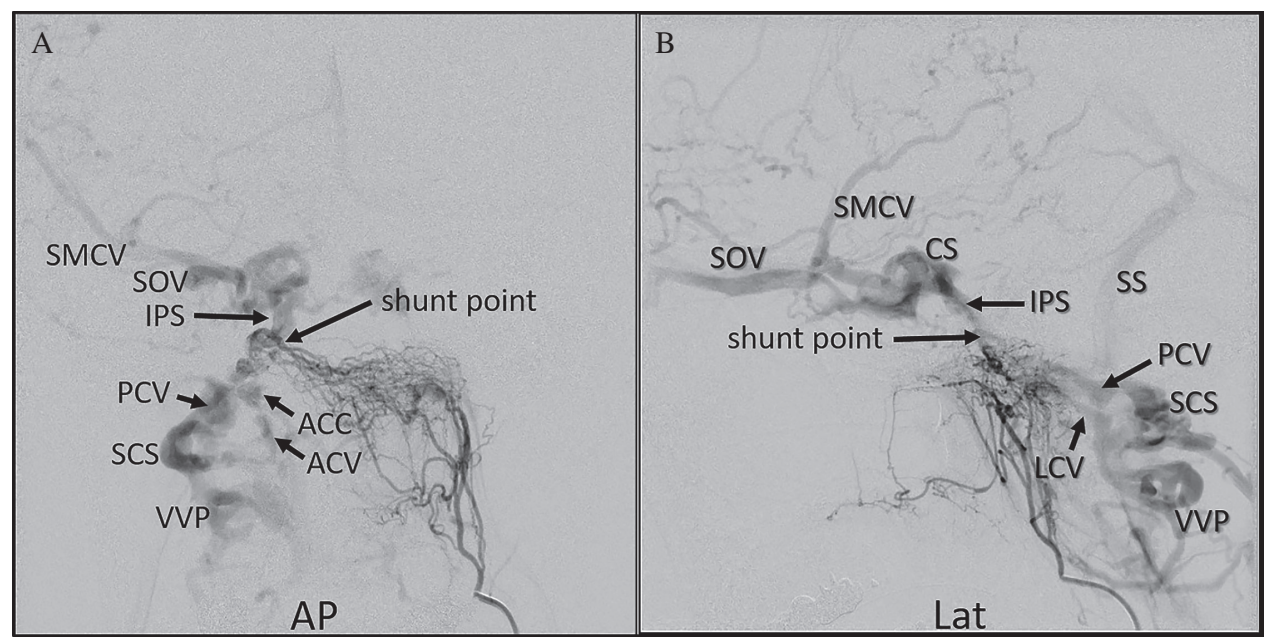

Fig. 2 Left ascending pharyngeal artery angiogram (A: AP view, B: lateral view). The left ascending pharyngeal artery angiogram shows DAVF located just ventrolateral to the right IPS. Since the proximal side of the right IPS is obstructed, the main venous drainage flow refluxes retrogradely from the IPS to the CS. ACC: anterior condylar confluence; ACV: anterior condylar vein; CS: cavernous sinus; DAVF: dural arteriovenous fistula; IPS: inferior petrosal sinus; LCV: lateral condylar vein; PCV: posterior condylar vein; SCS: suboccipital cavernous sinus; SMCV: superficial middle cerebral vein; SOV: superior ophthalmic vein; SS: sigmoid sinus; VVP: vertebral venous plexus

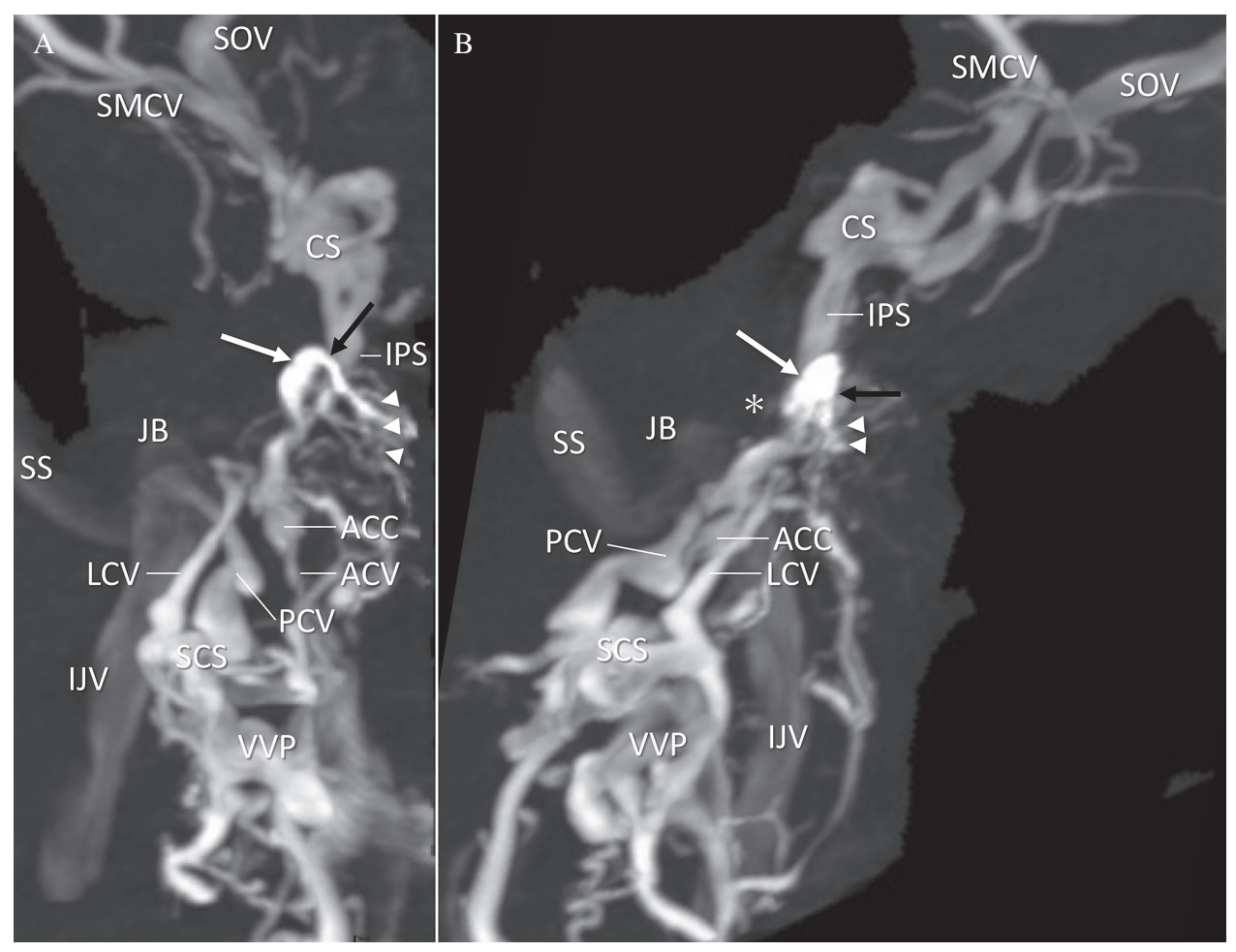

Fig. 3 3D left ascending pharyngeal artery angiogram (A: AP view, B: lateral view). The shunt point is located at the IPCV (black arrows). The white arrows show the venous pouch in the IPCV, and the white arrow heads show the feeders from the ascending pharyngeal artery. The asterisk shows the occluded right IPS. ACC: anterior condylar confluence; ACV: anterior condylar vein; CS: cavernous sinus; IJV: internal jugular vein; IPCV: inferior petroclival vein; IPS: inferior petrosal sinus; JB: jugular bulb; LCV: lateral condylar vein; PCV: posterior condylar vein; SCS: suboccipital cavernous sinus; SMCV: superficial middle cerebral vein; SOV: superior ophthalmic vein; SS: sigmoid sinus; VVP: vertebral venous plexus 
proximal side of the ACC were also densely visualized (Figs. 2 and 3).

Based on the above findings, although it is very rare, anatomically, the shunt point was considered to be located in the IPCV.

\section{Treatment}

We decided to perform selective coil embolization of the shunt point and venous pouch over the reflux to the IPS. A 6Fr long sheath: Radifocus Introducer II H was placed in the left femoral vein for approaching transvenously. A 4Fr CX catheter was placed selectively in the left APA through the right femoral artery for intraoperative angiography.

First, 6Fr Launcher (Medtronic, Minneapolis, MN, USA) was guided into the internal jugular vein (IJV) as a guiding catheter using Radifocus guidewire 0.035 (Terumo Corporation) coaxially with the 4Fr CX catheter. The proximal side of the right IPS was obstructed, but the distance between the IJV and obstructed region of the IPS was short so that the catheter could be passed through the obstructed IPS from the proximal side of the IPS using Radifocus guidewire 0.035 . The 6Fr Launcher could be guided to and placed in the proximal region of the IPS, and the 4Fr CX catheter was removed.

Then, Echelon microcatheter (Medtronic, Irvine, CA, USA) was passed through the 6Fr Launcher and guided into the CS through the IPS using Radifocus GT wire $0.01290^{\circ}$ (Terumo Corporation). Headway 17 microcatheter (MicroVention, Aliso Viejo, CA, USA) was also passed through the 6Fr Launcher and guided into the venous pouch immediately after the shunt point through the IPS using ASAHI CHIKAI 0.014 (Asahi Intecc, Aichi, Japan). It was planned to embolize the shunt point and venous pouch mainly using Headway 17, and when residual reflux from the IPS into the CS remains, the distal side of the IPS is to be embolized through the Echelon (Fig. 4A).

Through the Headway 17, the shunt point over the venous pouch and then outlet of the IPS were embolized sequentially with HyperSoft 3D $4 \times 80 \mathrm{~mm}$ (MicroVention) $\times 2$, Orbit Galaxy Complex XS $4 \times 80 \mathrm{~mm}($ CERENOVUS, Irvine, CA, USA) $\times 3$, Orbit Galaxy Complex Fill $4 \times 120 \mathrm{~mm} \times 2$, HyperSoft 3D $3.5 \times 80 \mathrm{~mm}$, HyperSoft 3D $2.5 \times 60 \mathrm{~mm} \times 2$, Orbit Galaxy Helical XS $2 \times 80 \mathrm{~mm}$ $\times 2$, Orbit Galaxy Complex XS $3.5 \times 75 \mathrm{~mm} \times 2$, HyperSoft 3D $3.5 \times 80 \mathrm{~mm}$, HyperSoft 3D $2.5 \times 60 \mathrm{~mm}$, and HyperSoft

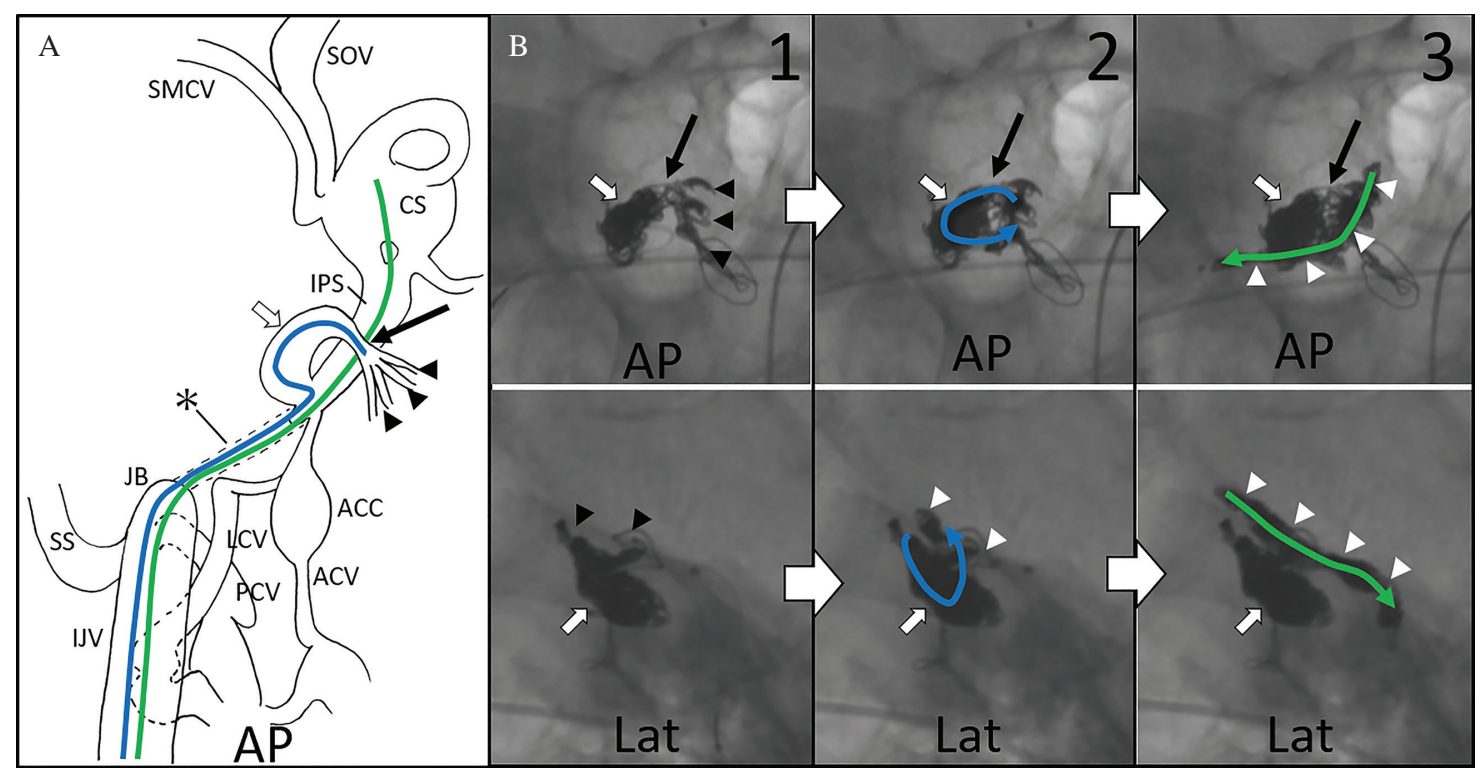

Fig. 4 The schema of our treatment strategy $(\mathbf{A})$ and the X-ray images of the coil mass in the time course of our procedure $(\mathbf{B})$. We performed TVE by the double catheter technique through the right IPS. The shunt point and the venous pouch in the IPCV were embolized through the Headway 17 microcatheter (blue line and arrow), and the IPS was embolized through the Echelon microcatheter (green line and arrow). The black arrows show the shunt point and the closed white arrows show the venous pouch in the IPCV. The black arrowheads show the feeders from the ascending pharyngeal artery, and the white arrowheads show the coil mass in the IPS. The asterisk shows the occluded right IPS. ACC: anterior condylar confluence; ACV: anterior condylar vein; CS: cavernous sinus; IJV: internal jugular vein; IPCV: inferior petroclival vein; IPS: inferior petrosal sinus; JB: jugular bulb; LCV: lateral condylar vein; PCV: posterior condylar vein; SMCV: superficial middle cerebral vein; SOV: superior ophthalmic vein; SS: sigmoid sinus; TVE: transvenous coil embolization; Echelon microcatheter: Medtronic, Irvine, CA, USA; Headway 17 microcatheter; MicroVention, Aliso Viejo, CA, USA 
3D $3 \times 60 \mathrm{~mm}$. Since reflux from the IPS to the CS slightly remained at this point, the IPS was obstructed with Orbit Galaxy Helical XS $2 \times 60 \mathrm{~mm}$ and Orbit Galaxy Helical $\mathrm{XS} 2 \times 80 \mathrm{~mm} \times 2$ through Echelon, which completely disappeared reflux from the IPS to the CS, SOV, and SMCV, and treatment was completed (Fig. 4B).

The postoperative course was favorable, no new neurological deficit developed, and right oculomotor nerve palsy was improved. Pulsatile tinnitus, ophthalmalgia, blepharedema, and chemosis noted before treatment also disappeared by the time of discharge. DAVF had completely disappeared on MRI and MRA at 1.5 months after treatment and symptoms were also resolved. As of 6 months after treatment, no recurrence has occurred.

\section{Discussion}

Reportedly, DAVF accounts for about $10 \%-15 \%$ of cases of intracranial vascular malformation. ${ }^{4-6)}$ In a survey in Japan, the annual incidence (detection rate) was 0.29 in 100000 people. . $^{7}$

The arterial and venous vascular anatomy is very complex in DAVF, and the close preoperative investigation is very important to decide on a treatment strategy. The venous structure is diverse and complex especially in DAVF around the ACC in the posterior fossa. DAVF around the ACC has recently been increasingly reported with recent improvement of the diagnostic ability of 3D imaging, such as 3D-CTA and 3D-DSA, and enlightenment of case
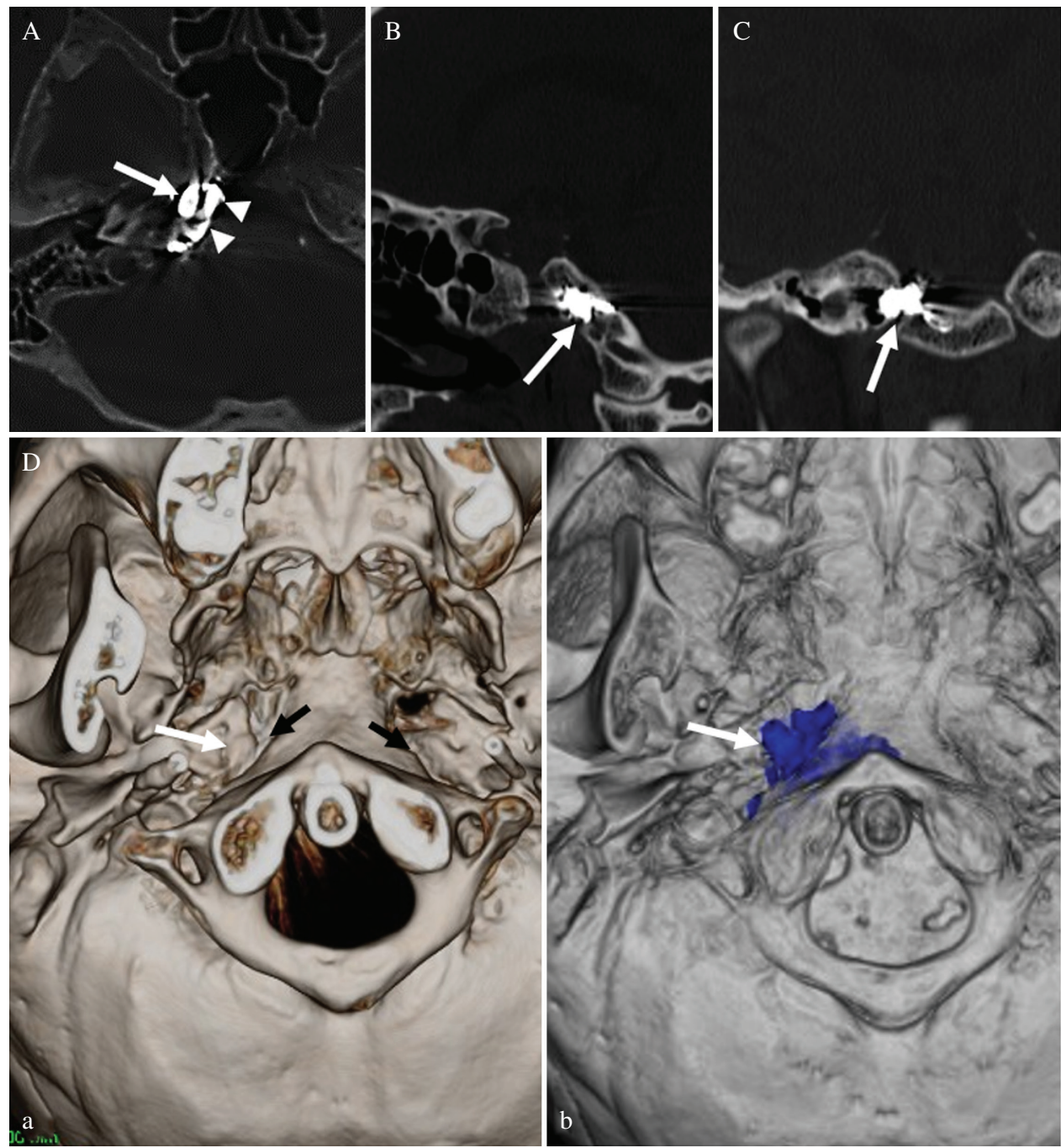

Fig. 5 The CT after treatment (A: axial view, B: sagittal view, C: coronal view, D: extra cranial 3D image of the skull base). The CT after embolization demonstrates the coil mass in the IPCV (white allows) at the extracranial surface of the petroclival fissure (black arrows) and in the IPS (arrowheads). The blue area in (D-b) shows the coil mass with higher CT number than the skull base bone. IPCV: inferior petroclival vein; IPS: inferior petrosal sinus 
reports, ${ }^{7)}$ but DAVF of the ACC still accounts for only about $3.6 \%-5.1 \%$ of all DAVF cases ${ }^{5,6,8)}$ and $5.0 \%$ in a survey in Japan, ${ }^{7)}$ showing that it is a rare disease.

Veins flowing into the ACC from the CS include the IPCV distributed on the inferior (extracranial) surface of the PCF. It is located at a mirror-image of the IPS distributed on the superior (intracranial) surface of the PCF, and these communicate with each other., ${ }^{1,2)}$ The IPCV is normally very small vein, but it dilates when the venous pressure rises due to an intracranial shunt lesion and it may serve as an access route to the CS, being a vein to be aware of. ${ }^{1)}$ The present case may have been DAVF with a shunt in the IPCV. To our knowledge, only one case of DAVF with a shunt in this region has previously been reported, indicating that this is a very rare shunting region. ${ }^{9}$ On postoperative cone beam CT, the coil mass was demonstrated continuously from the extracranial IPCV to the intracranial IPS. The presence of a shunting region in the IPCV was confirmed (Fig. 5) based on the intraoperative findings combined with the detailed findings of multi-planer reconstruction (MPR) prepared by rotational angiography presented in a book written by Kiyosue et al. ${ }^{10}$

Pulsatile tinnitus is observed in many cases of DAVF of the ACC and that located around the hypoglossal canal, and symptoms, such as hypoglossal nerve palsy, have been reported, ${ }^{5,6,11)}$ but obstruction of the proximal side of the IPS may cause reflux into the CS, SOV, and SMCV and mainly induce ocular symptoms similar to those of DAVF of the CS, to which attention should be paid., ${ }^{4,5,9,11-15)}$ The present case was similar to a previous case report in which blood flow refluxed in the IPS into the CS due to obstruction of the main venous drainage route, the proximal side of the IPS, and further reflux into the SOV mainly induced ocular symptoms similar to those of DAVF of the CS.

For treatment, TVE (occasionally, transarterial embolization [TAE] and TAE + TVE) is mainly performed. . $^{5-8,11,13,15,16)}$ Since the vein distribution is complex, ${ }^{17)}$ to accurately identify the shunt point and vein distribution, it is important to carefully investigate a treatment strategy and access route by making full use of 3D-DSA and understanding the 3D vascular anatomy of each case. ${ }^{11,14)}$ Treatment by TVE retrogradely through the $\mathrm{SOV}^{18)}$ and TVE using Onyx as an embolic material ${ }^{8)}$ have also been reported. We also succeeded in curing the patient by TVE. The shunt point and continuous venous pouch were embolized with a minimally necessary coils and slightly remaining reflux into the IPS was embolized by adding coils through another microcatheter placed on the distal side of the IPS beforehand, and safe and favorable embolization was achieved.
In addition, we could understand the detailed anatomy of the shunt point by selective 3D-DSA of the left APA on the healthy side. The densest region of contrast medium is considered the shunt point, ${ }^{3)}$ and the 3D-DSA findings were useful also for the present case. Imaging of the ipsilateral external carotid artery may overlap the lesion and make it unclear, but the vein and artery do not overlap on imaging from the contralateral APA and the image provides the minimum necessary information useful to decide on a treatment strategy for DAVF.11,14)

\section{Conclusion}

Treatment of a very rare case of DAVF including the IPCV was reported. TVE was performed through the IPS and achieved favorable embolization and outcome. To accurately identify the shunt point and venous drainage structure, it may be important to understand the 3D vascular anatomy in each case by making full use of 3D-DSA.

\section{Disclosure Statement}

None of the first and co-authors have any conflicts of interest.

\section{References}

1) Katsuta T, Matsushima T, Uda K: [Surgical anatomy of the skullbase venous system: petroclival region.] Jpn J Neurosurg (Tokyo) 2008; 17: 738-744. (in Japanese)

2) Tanoue S, Kiyosue H, Sagara $Y$, et al: Venous structures at the craniocervical junction: anatomical variations evaluated by multidetector row CT. Br J Radiol 2010; 83: 831-840.

3) Satow T, Murao K, Matsushige T, et al: Superselective shunt occlusion for the treatment of cavernous sinus dural arteriovenous fistulae. Neurosurgery 2013; 73: ons100-ons105.

4) Barnwell SL, Halbach VV, Dowd CF, et al: Dural arteriovenous fistulas involving the inferior petrosal sinus: angiographic findings in six patients. AJNR Am J Neuroradiol 1990; 11: 511-516.

5) Hsu YH, Lee CW, Liu HM, et al: Endovascular treatment and computed imaging follow-up of 14 anterior condylar dural arteriovenous fistulas. Interv Neuroradiol 2014; 20: 368-377.

6) Spittau B, Millán DS, El-Sherifi S, et al: Dural arteriovenous fistulas of the hypoglossal canal: systematic review on imaging anatomy, clinical findings, and endovascular management. J Neurosurg 2015; 122: 883-903.

7) Kuwayama N, Kubo M, Endo S, et al: [Present status in the treatment of dural arteriovenous fistulas in Japan.] Jpn J Neurosurg (Tokyo) 2011; 20: 12-19. (in Japanese) 
8) Takemoto K, Tateshima S, Rastogi S, et al: Onyx embolization of anterior condylar confluence dural arteriovenous fistula. J Neurointerv Surg 2014; 6: e13.

9) Sugiura Y, Nishizawa S: Arteriovenous fistula involving the inferior petroclival vein-case report. Neurol Med Chir (Tokyo) 2011; 51: 45-47.

10) Kiyosue H: [Kekkannaichiryo no tame no Kekkankaibo Nojomyaku]. 1st ed. Gakken Medical Shujunsha. Tokyo, 2016; 155-164. (in Japanese)

11) Miyachi S, Ohshima T, Izumi $T$, et al: Dural arteriovenous fistula at the anterior condylar confluence. Interv Neuroradiol 2008; 14: 303-311.

12) Yamada M, Miyasaka $Y$, Kitahara $Y$, et al: Dural arteriovenous malformation involving the inferior petrosal sinuscase report. Neurol Med Chir (Tokyo) 1994; 34: 300-303.

13) Kato S, Fujii M, Tominaga T, et al: [A case of dural arteriovenous fistula of the inferior petrosal sinus successfully treated by transarterial and transvenous embolizations.] No Shinkei Geka 2002; 30: 981-984. (in Japanese)
14) Sasaki $T$, Nagashima $H$, Satoh $D$, et al: Dural arteriovenous fistula around the anterior condylar confluent: report of two cases. JNET 2008; 2: 212-216.

15) Ihoshi $S$, Miyata $K$, Sugino $T$, et al: [Ocular symptoms associated with a dural arteriovenous fistula of the anterior condylar confluence: case report.] Surg Cereb Stroke 2013; 41: 285-289. (in Japanese)

16) Ernst R, Bulas R, Tomsick $T$, et al: Three cases of dural arteriovenous fistula of the anterior condylar vein within the hypoglossal canal. AJNR Am J Neuroradiol 1990; 20: 2016-2020.

17) San Millán Ruíz D, Gailloud $P$, Rüfenacht $D A$, et al: The craniocervical venous system in relation to cerebral venous drainage. AJNR Am J Neuroradiol 2002; 23: 1500-1508.

18) Gentric JC, Ferré JC, Raoult H, et al: Endovascular treatment of inferior petrosal sinus dural arteriovenous fistula via percutaneous puncture of the superior ophthalmic vein. J Neuroradiol 2013; 40: 144-146. 